\title{
Junior doctors' communication with hospital pharmacists about prescribing: findings from a qualitative interview study
}

\author{
David Rhys Axon, ${ }^{1}$ Rosemary Hwee Mei Lim, ${ }^{2}$ Penny J Lewis, ${ }^{3}$ Sarena Sandher, ${ }^{2}$ \\ Jenna Thondee, ${ }^{2}$ Karen Edwards, ${ }^{4}$ Rachel L Howard ${ }^{2}$
}

'Department of Pharmacy Practice and Science, The University of Arizona College of Pharmacy, Tucson, Arizona, USA

${ }^{2}$ Reading School of Pharmacy, University of Reading, Reading, UK

${ }^{3}$ Division of Pharmacy and Optometry, School of Health Sciences, The University of Manchester, Manchester, UK ${ }^{4}$ Frimley Health NHS Foundation Trust, Frimley, UK

\section{Correspondence to}

Dr Rosemary Hwee Mei Lim; r. h.m.lim@reading.ac.uk

Received 13 November 2017 Revised 14 January 2018 Accepted 16 January 2018

Published Online First

6 February 2018

EAHP Statement 5: Patient Safety and Quality Assurance.
Check for updates

To cite: Axon DR, Lim RHM, Lewis PJ, et al.

Eur J Hosp Pharm

2018:25:257-261.

\begin{abstract}
Objectives To explore factors affecting communication between Foundation Year (FY) 1 doctors and hospital pharmacists about prescribing from the junior doctors' perspective.

Methods Trained interviewers $(n=4)$ conducted semistructured interviews with FY1 doctors who were purposively sampled from three hospitals in England. FY1 doctors were asked about their experiences of communication with hospital pharmacists about their prescribing; instances where they disagreed with or did not implement a hospital pharmacist's recommendation; and their preferences for communicating with hospital pharmacists about prescribing. Interviews were audiorecorded, transcribed verbatim and analysed thematically.
\end{abstract}

Results A total of $27 \mathrm{FY} 1$ doctors were interviewed. Findings were categorised into four main themes: (1) nature and context of communication; (2) FY1 doctors' perceptions of communication with hospital pharmacists; (3) factors influencing FY1 doctors' decision whether to act on pharmacists' prescribing recommendations; and (4) suggestions to improve communication with pharmacists. FY1 doctors and hospital pharmacists generally communicated well. FY1 doctors appreciated and frequently acted on pharmacists' advice yet there was deference to senior medical staff when advice differed. Joint ward rounds, pharmacist-led teaching sessions and a standardised approach to communication were all suggested as ways to improve communication and may increase the likelihood of pharmacists' recommendations being acted on.

Conclusions FY1 doctors and hospital pharmacists communicated frequently about medication prescribing. Issues occurred when there were differences in professional judgement between senior medical staff and pharmacists but these were usually resolved satisfactorily for the FY1 doctor. Further interventions to improve communication and safe prescribing could involve a multidisciplinary and systems approach.

\section{INTRODUCTION}

Communication problems between healthcare professionals (HCPs), such as doctors and pharmacists, are prevalent and known to contribute to medication errors. ${ }^{1}$ In particular, poor communication has been identified as one factor that can affect prescribing errors. ${ }^{2}$ Studies have already explored communication between doctors and pharmacists in primary care from both professionals' perspectives, ${ }^{3-6}$ but there is a lack of research investigating communication between doctors and pharmacists in secondary care, ${ }^{7}$ particularly from the junior (Foundation Year (FY) 1) doctors' (doctors in their first year of training postgraduation) perspective with whom pharmacists have frequent contact.

Studies in primary care settings in several countries have shown that doctors act on pharmacist's recommendations $46 \%-100 \%$ of the time (median $79 \%),{ }^{8-12}$ but it is unknown why this variation exists and why doctors do not act on pharmacists' recommendations. These findings suggest that there may be unaddressed issues with communication that could be improved. Primary care research found that doctors' negative attitudes towards pharmacists $^{34}$ and their lack of appreciation for pharmacists' skills ${ }^{56}$ can cause communication problems, for example, no feedback following pharmacists' recommendations and a disinterest in collaboration from doctors. Poor communication between FY1 doctors and hospital pharmacists has also been identified as a barrier to effective feedback. ${ }^{13}$

A previous study found that FY1 doctors made the most prescribing errors, but since they are generally responsible for the majority of prescribing it could not be assumed that they make more prescribing errors than senior doctors (registrars and consultants). ${ }^{14}$ A more recent study found that FY1 doctors were twice as likely to make a prescribing error compared with consultants. ${ }^{15}$ FY1 doctors have previously said that one-way communication with little discussion and few opportunities for learning contributed to prescribing errors, ${ }^{2}$ suggesting better communication may reduce prescribing errors and improve medication safety.

Exploring FY1 doctors' views of communication with hospital pharmacists is important to further add to the knowledge base of communication between doctors and pharmacists. Identifying factors that facilitate or hinder communication could help inform strategies to reduce prescribing errors and improve pharmaceutical care.

Therefore, the aim of this study was to explore factors affecting communication between FY1 doctors and hospital pharmacists about prescribing issues from the FY1 doctors' perspective.

\section{METHODS}

\section{Study design}

This study used semistructured interviews with FY1 doctors in a secondary care setting to elicit information about their communication with 


\section{Box 1 Interview questions used in the study}

1. Can you tell me about the last three conversations you had with a pharmacist about your prescribing?

- Who started the conversation?

- What was the conversation was about?

- What was particularly good or helpful about this conversation?

- What would have improved the conversation (on the part of the doctor or the pharmacist)?

2. Can you think of an instance where a pharmacist has recommended a change in medication that you disagreed with or did not implement?

-What was the recommendation?

- How was the recommendation made (written note, conversation, in medical notes, etc)?

- Why did you disagree with the recommendation?

Why did you choose not to implement the recommendation?

- Was the rationale for this decision discussed with the pharmacist (why/why not)?

3. When a pharmacist gives you advice about (or queries) your prescribing, do you prefer them to talk to you about it, or to write it down for you?

-Why is this form of communication preferred?

- If written, where would you prefer it to be written?

- If verbal, do you prefer face to face or telephone communication?

hospital pharmacists about their prescribing. The semistructured approach allowed participants to openly discuss their experiences of communication with hospital pharmacists, while retaining emphasis on the areas of interest.

\section{Development of interview schedule}

Interview questions were developed from relevant literature and with discussion between the interprofessional research team (who consisted of pharmacists and doctors with extensive experience of medication errors and their causes). Three core questions were developed, with prompts to encourage discussion: the first question asked about FY1 doctors' last three conversations with hospital pharmacists about their prescribing, the second question asked about instances where the FY1 doctor disagreed with or did not implement a hospital pharmacist's recommendation and the final question asked about FY1 doctors' preferences for communicating with hospital pharmacists about their prescribing. Questions deliberately avoided asking directly about communication problems to allow doctors to identify what they perceived to be problems and to avoid talking specifically about prescribing errors. The questions were tested in a small pilot study of FY1 doctors and did not identify any issues; thus, no changes were made to the questions for this study. The interview questions used in this study are summarised in box 1 .

\section{Recruitment of participants}

In order to participate in this study, participants had to be an FY1 doctor working at a hospital in England. A convenience sample of FY1 doctors was identified via existing contacts at three acute hospital trusts in England (Oxford, Reading and Manchester). These doctors were sent information letters via email inviting them to participate in the study. Members of the research team also recruited FY1 doctors by attending FY1 doctor training days. Interviews were conducted with all participants who agreed to participate in the study until data saturation was reached. Written informed consent was sought from participants prior to data collection.

\section{Data collection}

Four researchers (preregistration pharmacists and undergraduate pharmacy students) who had received appropriate training conducted one-on-one audiorecorded interviews $(n=27)$ between November 2012 and June 2013. Interviews were conducted in-person at the hospital site or via teleconferencing facilities at the University according to the preference of the FY1 doctor being interviewed. Interviewers introduced themselves and their role (pharmacy student or preregistration pharmacist) at the start of the interview.

\section{Data analysis}

Two researchers transcribed interview recordings verbatim. Another independent researcher analysed the data using thematic analysis aided by QSR International's NVivo V.10 (2012) qualitative data management software. Data were coded, and similar codes grouped into categories to identify themes. The analysis process was iterative and the researcher referred back to previous points as new observations became apparent. Another trained researcher independently coded a third (nine) of the interview transcripts. On comparison, the two code lists provided a good match with $94 \%$ agreement. Minor differences were discussed between the two researchers until agreement was reached.

\section{RESULTS}

\section{Participant demographics}

A total of 27 participants across the three hospital sites were recruited and interviewed. All FY1 doctors who responded were interviewed. Participants worked in general medicine $(n=15)$, general surgery $(n=9)$ and unknown $(n=3)$ settings at the time of the interview. Participants' time in their current post varied from a few days to four and a half months so some participants drew on their previous experiences working in other settings when interviewed. Interviews lasted between 4 and $16 \mathrm{~min}$.

\section{Themes identified}

Thematic analysis identified four main themes described below: (1) nature and context of communication; (2) perceptions of communication with hospital pharmacists; (3) factors influencing doctors' decision whether to act on pharmacists' recommendations; and (4) suggestions to improve communication with pharmacists.

\section{Nature and context of communication}

Both FY1 doctors and hospital pharmacists initiated communication about prescribing. FY1 doctors usually approached pharmacists before prescribing for advice such as interpreting guidelines or calculating doses, while hospital pharmacists contacted doctors after medications were prescribed or after reviewing patients' blood test results, for example to suggest an alternative medicine or dose. Many FY1 doctors said they had regular contact with hospital pharmacists, while only a couple reported few or intermittent communications with hospital pharmacists, usually when the doctor was working nights. Reported frequency of communication with hospital pharmacists varied 
depending on the time of day, with fewer interactions occurring out of hours (evenings, nights and weekends):

We've got a pharmacist who comes quite regularly, almost everyday in the mornings. (I11)

The doctors' specialty also affected communication frequency, for example doctors on a medical rotation reported communicating more frequently with hospital pharmacists compared with those on a surgical rotation. FY1 doctors reported that they communicated most frequently with hospital pharmacists in their first post, and that this contact reduced as they became more experienced:

I found the pharmacists really useful on stroke especially at the beginning, because when you're new you don't know your doses. (I18)

\section{Perceptions of communication with hospital pharmacists}

FY1 doctors described pharmacists positively and commented that pharmacists were helpful, had excellent knowledge of medicines, always willing to answer questions and approachable:

Pharmacists that I've worked with ... have been fantastic and really approachable, happy to have questions, don't make you feel stupid when you don't know things and a really valuable resource. (I6)

Participants described how they worked closely with hospital pharmacists and many commented positively on their interactions describing communication using words such as 'clear', 'helpful' and 'pleasant'. FY1 doctors also commented that knowing the names of pharmacists aided communication by getting to know the pharmacists better and establishing rapport within the multidisciplinary team, which ultimately helped doctors' understanding of pharmacists' recommendations. It was apparent from the interviews that pharmacists provided FY1 doctors with a safety net for their prescribing and that this provided reassurance to these newly qualified prescribers:

I feel like they [the pharmacists] are a really nice safety check...I am comforted knowing it's there. (I3)

Conversely, some FY1 doctors provided examples of unhelpful communication. FY1 doctors expressed how communication with pharmacists could sometimes become frustrating, inconvenient and repetitive (especially true if several pharmacists were involved in a prescribing scenario), and that communication with pharmacists may not be a priority when they have other tasks to complete:

You're trying to do an important job and your eleventh bleep is from a pharmacist ... sometimes in the context of lots of other demands made on you it would be nice not to have that extra bleep. (I4)

One participant also raised timing of communication by pharmacists as an issue:

He takes quite un-opportune times to talk to you ... it's just a timing issue. (I13)

Despite these negative comments, doctors acknowledged the information given by pharmacists may be useful in the future or to someone else.

\section{Factors influencing doctors' decision whether to act on pharmacists' recommendations}

FY1 doctors described how they would discuss pharmacists' recommendations one to one to ensure that they understood the advice and to resolve any misunderstandings. FY1 doctors
Box 2 Suggestions to improve communication between

junior doctors and pharmacists

Greater access to pharmacists

- Joint ward rounds

- Guidelines review

- Pharmacist teaching sessions

- Standardised communication methods

appreciated and generally agreed with pharmacists' prescribing advice, accepting their recommendations. On other occasions, FYI doctors came to a mutual agreement over the correct course of action with the pharmacist:

I thought it was very important to discuss these issues, especially if there is a risk of prescribing error. (I19)

The trust that doctors placed in pharmacists prompted doctors to act on their recommendations and facilitated two-way communication:

Nice for the pharmacist to pick that up ... I completely trust pharmacists. (I23)

Those occasions in which FY1 doctors did not act on pharmacists' recommendations were explored during the interviews. It emerged that a divergence between senior doctors' and pharmacists' professional opinions would commonly lead to a deference to the opinion of senior doctors; senior doctors were more experienced and FY1 doctors did not wish to challenge their decisions:

They [the consultants] have more wisdom than I do so I usually take their advice. (I3).

Additionally, there were a small number of occasions in which FY1 doctors did not act on pharmacists' recommendations:

They've suggested 'why don't we try this' and we've said 'well actually no we're not doing that because of $x, y, z$ '. (I6)

\section{Suggestions to improve communication with pharmacists}

FY1 doctors made suggestions for how pharmacists could improve the likelihood of their recommendations being acted on, as summarised in box 2 .

FY1 doctors suggested that greater access to pharmacists would be helpful, especially out of regular working hours, as they felt pharmacists could be difficult to find, pharmacy departments could be slow and their phones always busy. FY1 doctors indicated the lack of continuity of staff to be an issue and preferred a dedicated, regular ward pharmacist who could be approached for advice when necessary, which would reduce repetitive, time-consuming conversations caused by different pharmacists' covering ward rounds:

It would be better if the ward pharmacists were more ward based so they were always there ... at the moment it's a bit sporadic when you see them. (I13)

Some FY1 doctors outlined the benefits of having pharmacists on ward rounds, an existing practice in some hospitals that enables pharmacists to review patients' medications together as part of a multidisciplinary team:

I've seen in other hospitals and from past experience, the pharmacists actually do ward rounds with the consultant as an extra member of the team. I think that's much better and a very sensible system. (I9) 
FY1 doctors in the study raised concerns about guidelines and protocols that were difficult to interpret or contained conflicting information. In addition, it was felt that there was a lack of protocols, for example, in the administration of medicines. It was suggested that unclear guidelines and protocols could be reviewed to improve the decision-making process and address FY1 doctors' concerns:

Our department doesn't have a protocol for one medication that we commonly use ... from our point of view if we could sort out a departmental protocol it would be even more helpful and would solve all of these problems. (I9)

FY1 doctors would welcome more pharmacist-led teaching sessions on basic prescribing skills such as how to prescribe on a medicine chart or how to calculate values such as creatinine clearance:

I think that teaching session was really good. So more of them. (I23)

Data suggest that implementing a consistent communication method between hospital pharmacists and FY1 doctors could increase the likelihood of pharmacists' recommendations being implemented by FY1 doctors. Some doctors preferred verbal communication, others written communication and some a combination of both verbal and written communication. However, there was no unanimous agreement over which was preferred.

\section{DISCUSSION}

Our study found that FY1 doctors communicate well with hospital pharmacists but suggested that communication between hospital pharmacists and senior doctors was less collaborative. Hospital pharmacists' input was generally appreciated and their prescribing recommendations acted on, except some instances where FY1 doctors acted on senior doctors' advice instead. Suggestions for improving communication include greater access to pharmacists, joint ward rounds and more pharmacist-led teaching sessions.

FY1 doctors described several positive aspects of communication between themselves and hospital pharmacists. Trusting and knowing each other has been found to improve communication between doctors and pharmacists in primary care, ${ }^{341617}$ and the findings of our study suggest that this may also be the case in secondary care since FY1 doctors preferred to work with regular ward pharmacists who they could become acquainted with, rather than irregular or part-time pharmacists. However, when faced with different advice FY1 doctors would usually defer to senior doctors' recommendations rather than the pharmacists', which may be because doctors work in a hierarchical structure and FY1 doctors do not want to upset the team relationship. ${ }^{18}$ Further work could explore the role of trust in decision-making and communication between HCPs.

FY1 doctors said that pharmacists ensured effective and safe prescribing, an important finding that concurs with a previous study and demonstrates the impact of pharmacists' skills. ${ }^{19}$ FY1 doctors in this study valued and were confident to act on pharmacists' prescribing recommendations or mutually agree an acceptable course of action. However, FY1 doctors said that some senior doctors would continue to act on their own experience, rather than the pharmacists' recommendations.

Our study found that doctors usually discussed pharmacists' prescribing recommendations, which supports the findings of a recent study that found $85 \%$ of issues identified by pharmacists were discussed with the doctor and the rest were discussed with nurses or medical students. ${ }^{20}$ Discussions with HCPs other than doctors may explain why conversations between pharmacists and doctors did not occur in some instances and account for why pharmacists' recommendations were not acted on.

Our study found that poor timing of pharmacy queries from pharmacists was a problem. Doctors and pharmacists have already agreed the need for greater collaboration to improve pharmaceutical care. ${ }^{21}$ Developing a relationship based on mutual understanding of competing priorities and agreeing on the most appropriate mode of communication and optimal timing of communication are important next steps. In addition, joint ward rounds could potentially reduce communication problems between FY1 doctors and hospital pharmacists, such as misinterpreting communication and difficulty contacting each other, as prescribing queries would be resolved at the time of prescribing. Joint ward rounds have already been shown to optimise decision-making, ${ }^{22}$ reduce medication errors ${ }^{23} 24$ and decrease medicine-related problems. ${ }^{25}$ However, joint ward rounds require both the doctor and the pharmacist to be available at the same time, which could prove challenging given that doctors' heavy workload has previously been identified as a barrier to communication. ${ }^{26}$

FY1 doctors appreciated pharmacists teaching basic skills such as how to prescribe, and would like more of this. Since the time of the study, there has been progress in this area; the Royal College of Physicians in the UK has introduced guidance that recommends prescribing induction should be practically focused and cover safety principles, and that junior doctors should receive regular feedback on prescribing errors in a structured and supportive way. ${ }^{27}$ Pharmacists teaching FY1 doctors in a hospital setting resulted in a $37.5 \%$ reduction in prescribing errors, ${ }^{28}$ which highlights the value of pharmacists' skills in improving pharmaceutical care. Previous studies have found that junior doctors acknowledged their lack of prescribing skills ${ }^{29}$ and only $38 \%{ }^{30}$ of FY1 doctors considered themselves competent to prescribe at graduation. This explains the desire for this service, although improvements in teaching and assessing prescribing competence at medical schools could reduce this need in the future. Since the time of the study, there has been technological advances in the way prescribing is undertaken in acute hospitals for example a switch from paper-based to electronic prescribing. Future studies could investigate whether such changes influence communication about prescribing.

There were some limitations of this study. First, three study sites were used which is more representative of the study population than a single site but not wholly representative of the entire population. Second, distinctions were not made between doctor specialties or the pharmacist's level of experience, although a wide sample of pharmacists from across the three hospitals should have captured differences in experience and communication skills. Comparing communication between different specialties of doctor may identify communication problems that are inherent to one group rather than all junior doctors. Third, interviews were short with some interviewees only detailing one example rather than several; despite this data saturation was achieved.

\section{CONCLUSION}

FY1 doctors and hospital pharmacists communicated frequently, and both approach each other for different reasons. The majority of FY1 doctors appreciated input from hospital pharmacists about their prescribing, but where the senior doctor and hospital pharmacists' recommendations differed, FY1 doctors would 
defer to the senior doctor's advice. FY1 doctors' suggestions to improve communication such as joint ward rounds and more pharmacist-led teaching sessions have the potential to decrease prescribing errors and improve medication safety. Agreement on the most appropriate mode and timing of communication are important next steps. Future development of interventions to reduce prescribing errors could take into account the multifactorial issues identified in this study.

\section{What this paper adds}

\section{What is already known on this subject}

- Communication problems between doctors and pharmacists exist, but there is a lack of information about communication between Foundation Year 1 (FY1) doctors and hospital pharmacists from the FY1 doctors' perspective.

- Poor communication between FY1 doctors and hospital pharmacists may lead to prescribing errors.

\section{What this study adds}

- FY1 doctors and hospital pharmacists communicate frequently about prescribing.

- FY1 doctors valued pharmacists input and usually acted on pharmacists' prescribing recommendations, unless the senior doctor had a different recommendation.

- Joint ward rounds, improving prescribing guidelines and more pharmacist-led teaching sessions could improve communication.

Contributors RLH, DRA, PJL and KE developed the interview protocol. RLH, PJL, KE, SS and JT helped recruit participants for the study and conducted the interviews. SS and JT transcribed the interviews. DRA and RHML analysed the data and drafted the initial report. All authors reviewed and approved the final report.

Competing interests None declared.

Ethics approval This study received ethical approval from the University of Reading Research Ethics Committee (ref 12/12).

Provenance and peer review Not commissioned; externally peer reviewed.

(c) European Association of Hospital Pharmacists (unless otherwise stated in the text of the article) 2018. All rights reserved. No commercial use is permitted unless otherwise expressly granted.

\section{REFERENCES}

1 Howard R, Avery A, Bissell P. Causes of preventable drug-related hospital admissions: a qualitative study. Qual Saf Health Care 2008;17:109-16.

2 Coombes ID, Stowasser DA, Coombes JA, et al. Why do interns make prescribing errors? A qualitative study. Med J Aust 2008;188:89-94.

3 Van C, Mitchell B, Krass I. General practitioner-pharmacist interactions in professional pharmacy services. J Interprof Care 2011;25:366-72.

4 Bradley F, Ashcroft DM, Noyce PR. Integration and differentiation: a conceptual model of general practitioner and community pharmacist collaboration. Res Social Adm Pharm 2012:8:36-46.

5 Pottie K, Haydt S, Farrell B, et al. Narrative reports to monitor and evaluate the integration of pharmacists into family practice settings. Ann Fam Med 2008:6:161-5
6 Farrell B, Pottie K, Woodend K, et al. Shifts in expectations: evaluating physicians perceptions as pharmacists become integrated into family practice. J Interprof Care 2010;24:80-9.

$7 \square$ ström K, Duggan C, Bates I. Developing a way to improve communication between healthcare professionals in secondary care. Pharmacy Education 2007;7:279-85.

8 Soendergaard B, Kirkeby B, Dinsen C, et al. Drug-related problems in general practice: results from a development project in Denmark. Pharm World Sci 2006;28:61-4.

9 Gable KN, Stunson MJ. Clinical pharmacist interventions on an assertive community treatment team. Community Ment Health J 2010;46:351-5.

10 Weber CA, Ernst ME, Sezate GS, et al. Pharmacist-physician comanagement of hypertension and reduction in 24-hour ambulatory blood pressures. Arch Intern Med 2010;170:1634-9.

11 Bryant LJ, Coster G, Gamble GD, et al. The General Practitioner-Pharmacist Collaboration (GPPC) study: a randomised controlled trial of clinical medication reviews in community pharmacy. Int J Pharm Pract 2011;19:94-105.

12 Tahaineh L, Albsoul-Younes A, Al-Ashqar E, et al. The role of clinical pharmacist on lipid control in dyslipidemic patients in North of Jordan. Int J Clin Pharm 2011:33:229-36

13 Bertels J, Almoudaris AM, Cortoos PJ, et al. Feedback on prescribing errors to junior doctors: exploring views, problems and preferred methods. Int I Clin Pharm 2013;35:332-8.

14 Dean $B$, Schachter $M$, Vincent $C$, et al. Prescribing errors in hospital inpatients: their incidence and clinical significance. Qual Saf Health Care 2002;11:340-4.

15 Ashcroft DM, Lewis PJ, Tully MP, et al. Prevalence, nature, severity and risk factors for prescribing errors in hospital inpatients: prospective study in 20 UK hospitals. Drug Saf 2015:38:833-43.

16 Snyder ME, Zillich AJ, Primack BA, et al. Exploring successful community pharmacistphysician collaborative working relationships using mixed methods. Res Social Adm Pharm 2010;6:307-23.

17 Chui MA, Stone JA, Odukoya OK, et al. Facilitating collaboration between pharmacists and physicians using an iterative interview process. J Am Pharm Assoc 2014:54:35-41.

18 Lewis PJ, Tully MP. Uncomfortable prescribing decisions in hospitals: the impact of teamwork. J $R$ Soc Med 2009;102:481-8.

19 Zargarzadeh AH, Jacob S, Klotz RS, et al. Clinical pharmacists and basic scientists: do patients and physicians need this collaboration? Int J Clin Pharm 2011;33:886-94.

20 Stemer G, Lemmens-Gruber R. The clinical pharmacist's contributions within the multidisciplinary patient care team of an intern nephrology ward. Int J Clin Pharm 2011:33:759-62.

21 Kelly DV, Bishop L, Young S, et al. Pharmacist and physician views on collaborative practice: findings from the community pharmaceutical care project. Can Pharm J 2013:146:218-26.

22 O'Hare JA. Anatomy of the ward round. Eur J Intern Med 2008;19:309-13

23 Scarsi KK, Fotis MA, Noskin GA. Pharmacist participation in medical rounds reduces medication errors. Am J Health Syst Pharm 2002;59:2089-92.

24 Kucukarslan SN, Peters M, Mlynarek M, et al. Pharmacists on rounding teams reduce preventable adverse drug events in hospital general medicine units. Arch Intern Med 2003:163:2014-8.

25 Amruso NA, O'Neal ML. Pharmacist and physician collaboration in the patient's home Ann Pharmacother 2004:38:1048-52.

26 Teinilä T, Kaunisvesi K, Airaksinen M. Primary care physicians' perceptions of medication errors and error prevention in cooperation with community pharmacists. Res Social Adm Pharm 2011:7:162-79.

27 Royal College of Physicians. Supporting junior doctors in safe prescribing. 2017 https://www.rcplondon.ac.uk/projects/outputs/supporting-junior-doctors-safeprescribing (accessed 20 Dec 2017)

28 Webbe D, Dhillon S, Roberts CM. Improving junior doctor prescribing - the positive impact of a pharmacist intervention. Pharm J 2007;278:136-9.

29 Lewis PJ, Ashcroft DM, Dornan T, et al. Exploring the causes of junior doctors' prescribing mistakes: a qualitative study. Br J Clin Pharmacol 2014;78:310-9.

30 Heaton A, Webb DJ, Maxwell SR. Undergraduate preparation for prescribing: the views of 2413 UK medical students and recent graduates. Br J Clin Pharmacol 2008:66:128-34. 